\title{
MEASUREMENT AND CONTROL OF THE VARIABILITY OF SCANNING PRESSURE TRANSDUCER MEASUREMENTS
}

\author{
David D. Kuhl ${ }^{*}$, Lockheed Martin \\ and \\ Joel L. Everhart ${ }^{\dagger}$, James B. Hallissy ${ }^{\ddagger}$ NASA Langley Research Center \\ Hampton, VA
}

\begin{abstract}
This paper describes the new wall pressure measurement system and data-quality monitoring software installed at $14 \times 22 \mathrm{Ft}$ subsonic tunnel at the NASA Langley Research Center. The monitoring software was developed to enable measurement and control of the variability of the reference pressures and approximately 400 tunnel wall pressure measurements. Variability of the system, based upon data acquired over a year of wind tunnel tests and calibrations, is presented. The level of variation of the wall pressure measurements is shown to be predictable.
\end{abstract}

\section{INTRODUCTION}

Nearly 400 wall pressure orifices have been installed in the $14 \times 22 \mathrm{Ft}$ Subsonic Tunnel at NASA Langley Research Center to provide pressure boundary condition data for wind tunnel wall interference correction codes and computational fluid dynamic simulations of the in-tunnel flow field. The wall pressure measurement system includes 384 scanning differential pressure transducers (0.36-psid range), a pressure calibration unit (PCU), and a pressure standard to calibrate the PCU.

The increment between the model-induced wall pressures and the tunnel free-stream static pressure can be extremely small, requiring accurate measurements for proper quantification. Rigorous monitoring of the measurement system variability is required to insure accuracy to enable verifiable data quality standards. However, analysis of the resulting process data can require overwhelming manpower resources. Implementing statistical process control (SPC) methods can formalize and standardize the analysis process, providing a structure that can be automated via computer software and resulting in simplified visual display of the results. The development of such a monitoring system is the topic of this paper.

The SPC control charts are used to assess the variability of the process and identify if it is in control. If the process is in control and hence predictable, then the normal process variability may be quantified. If the process is not in control and unpredictable, then SPC analysis will identify when external modifiers are acting upon the process.

The wall pressure system is divided up into three separate processes: 1) the calibration of the PCU with the pressure standard every six months, 2) the daily calibration of the wall pressure transducers with the PCU daily, and 3) the repeated tunnel wall pressure transducer measurements several times a year. Repeating tunnel measurements requires acquisition of multiple data sets acquired with the same artifact at the same tunnel conditions. Organizationally, the Section 2 of the paper describes the process setups, the Section 3 discusses the SPC methodology applied to these processes, and the Section 4 describes monitoring of the processes over time. Results are summarized in the concluding section.

\section{EXPERIMENTAL SETUP}

Each of the wall pressure system processes has its own unique setup designed to minimize the effects of inherent external modifiers which may increase the variations in the measurements. The following subsections describe the tunnel and instrumentation used, and then a brief description of the setup of the three processes is presented.

\footnotetext{
* Member, AIAA; Aeronautical Engineer, Langley Program Office.

† Senior Member AIAA; Research Engineer, Aerothermodynamics Branch, AAAC.

${ }^{*}$ Senior Member AIAA; Test Engineer, Research Facilities Branch, AAAC.
} 


\section{Tunnel and Instrumentation}

The $14 \times 22 \mathrm{Ft}$ subsonic tunnel has a test section $21.75 \mathrm{ft}$ wide by $14.50 \mathrm{ft}$ high by $40 \mathrm{ft}$ long. There are several open and closed wall, ceiling, and floor configurations available with the tunnel, however, for the purposes of this paper, only the fully closed geometry is considered. The test section is approximately sealed except for a boundary layer removal system located at the entrance to the test section on the floor. For the purposes of this paper the boundary layer removal system is assumed to be operating. The maximum tunnel test section dynamic pressure $(\mathrm{Q})$ is approximately $132 \mathrm{psf}$.

The 384 wall pressures are measured using six 0.36-psi full scale 64-port Electronic Pressure Scanners (ESP) modules manufactured by Pressure Systems Incorporated (PSI). These transducer modules are housed external to the test section on the ceiling of the tunnel. The modules are enclosed in heater boxes to keep them at a constant temperature of approximately $120^{\circ} \mathrm{F}$. The manufacturer's accuracy rating for the transducers is $\pm 0.1 \%$ full scale (FS).

The wall pressure transducers are calibrated with the Model 8433 PCU manufactured by PSI. The PCU has a 1 psid differential High Accuracy Silicon Standard (HASS) pressure transducer to measure the calibration pressures. The manufacturer rated accuracy of the HASS transducer is $0.02 \%$ FS. The PCU has internal temperature compensation as well as a self-zeroing process.

The pressure standard used to calibrate the PCU is a Ruska Autofloat Gas Piston Gauge Model 2465 with a range of 0.2 to $600 \mathrm{psia}$ with a manufacturer rated accuracy of $0.0010 \%$ of the reading. This instrument has a calibration traceable to the National Institute of Standards and Technology (NIST).

\section{Calibration of the PCU}

The PCU's are calibrated in a thermally controlled laboratory environment with the pressure standard. The pressure standard applies, and measures, twenty-five differential pressures across the PCU transducer, spanning its full scale range. The pressures are sequenced in ascending and descending order as shown in Figure 1. A perfect calibration will result in a slope $=1$ from a first-order least square fit of the pressure standard readings and the PCU output pressures. The calculated quantity from the calibration is a slope adjustment; the slope adjustment is (1l/slope). The calibration slope adjustment is input into the processing algorithm of the PCU.

\section{Calibration of the Pressure Transducers}

Unlike the laboratory-environment calibration of the PCU, the pressure transducers are calibrated in situ. In calibration mode, a common reference pressure and a common measurement pressure are simultaneously applied to all of the differential transducers by shifting a mechanical sliding block inside of the module.

The PCU applies five different pressures covering the full-scale range of the transducers; 0 psid, 0.18 psid, $0.36 \mathrm{psid},-0.18 \mathrm{psid}$ and $-0.36 \mathrm{psid}$, as shown in Figure 2. Each pressure transducer output is individually fit with a fourth order polynomial curve fit of the form

$$
\text { Press }=C_{0}+C_{1} \text { Volt }+C_{2} \text { Volt }^{2}+C_{3} \text { Volt }^{3}+C_{4} \text { Volt }^{4}
$$

where Volt is the transducer voltage measured at the applied pressure, Press, from the PCU. The coefficients are recorded and used for subsequent measurement calculations.

\section{Pressure Transducer Measurements}

The pressure transducers shift from calibration mode to measurement mode by moving the mechanical block so that the measurement sides of all of the transducers are connected to the test section wall orifices. Wall orifices are connected to 372 of the 384 transducers, the other 12 transducers are shunted with the reference pressure.

The wall orifices are distributed along 12 axial rows covering the ceiling and the two walls of the tunnel. Figure 3 shows the wall orifice row distribution as seen from a cross section of the tunnel test section. Each row contains 31 ports distributed over the entire 40 -foot test section length. The orifices are not distributed evenly along the test section, as can be seen in Figure 4, 5 and 6 . The staggered distribution was designed to provide a higher density of orifices around the two primary model positions in the tunnel; one in the middle of the upstream half of the test section the other in middle of the downstream half of the test section. ${ }^{1}$

Figure 7 shows a limited schematic of the reference pressure system from the orifices on the walls to the upstream stagnation probe. A dedicated PCU is used to supply the wall pressure transducers with a prescheduled reference pressure during normal operation of the tunnel. This allows smaller range gages to be used, thereby increasing measurement accuracy. The dedicated PCU applies a constant differential pressure between the wall pressure transducer reference ports and the tunnel total pressure. The tunnel total pressure is used because it is more stable during normal tunnel operations than the tunnel static pressure. In the lower 
right hand corner of Figure 7 is the schedule of reference pressures for a $Q$ sweep from 0 to $140 \mathrm{psf}$.

\section{STATISTICAL PROCESS CONTROL METHODS}

Appropriate SPC methods are tailored and applied to each of the wall pressure system processes. All of the processes require chronological charts with calculated control limits set using the standard methods of SPC. ${ }^{2.3}$ For all of the processes control limits are time history based. The following subsections discuss the differences in the SPC methods used with each process.

\section{Calibration of the PCU}

The PCU calibration slope adjustment is charted using individuals and moving range charts as presented in Figure 8. The values on the $\mathrm{Y}$-axis of the charts are in percent of full scale of the PCU pressure transducer (1psid). The chart parameter means are shown as solid lines and the control limits are shown as light gray lines.

The individuals control chart plots chronologically each slope adjustment data point. The moving range chart plots chronologically the absolute value of the difference between the previous slope adjustment and the current slope adjustment data point. Both the individuals and moving range control charts are used to monitor point-to-point variation.

The control limits for both the individuals and moving range charts correspond to three standard deviation limits: The estimate of the standard deviation of the individual measurements, $\hat{\sigma}_{x}$, is

$$
\hat{\sigma}_{x}=\frac{\overline{m R}}{1.128}
$$

where $\overline{m R}$ is the mean moving range calculated from the data. The control limit on the moving range chart is set as

$$
\text { Control Limit }=3.268 \cdot \overline{m R}
$$

There are rules that detect whether a process is in control. If all the points on the charts for both the Individuals and Moving Range charts satisfy these rules, then the process, over that time frame, is in control. If the process is in control then there are no significant external modifiers effecting the variation of the process and the normal process variability may be quantified.
The Western Electric Company (WEC) rules are an accepted means of analyzing control charts. ${ }^{3}$ These rules go beyond checking for points falling inside or outside of the three standard deviation control limits by checking for patterns of out of control behavior as well. Table 1 below lists the four WEC rules, the first three of which are based upon $0.3 \%$ probability of encountering an event in a normal distribution of

\begin{tabular}{|c|}
\hline $\begin{array}{l}\text { Two out of the last three points above }+2 \text { or below }-2 \\
\text { standard deviations. }\end{array}$ \\
\hline $\begin{array}{l}\text { Four out of the last five points above }+1 \text { or below }-1 \\
\text { standard deviations. }\end{array}$ \\
\hline $\begin{array}{l}\text { Eight consecutive points on one side of } \\
\text { line. }\end{array}$ \\
\hline
\end{tabular}
individual measurements.

Table 1. The Western Electric Company (WEC) rules.

All of the points in Figure 8 pass the WEC rules and the process is in control. The normal process variability can be calculated when a process is in control. The normal process variability of the PCU calibration slope adjustment, reported as standard deviation, is $0.045 \%$ FS. The PCU's pressure transducer is specified by the manufacturer to be accurate within $0.02 \%$ FS. The slope adjustment process variability is twice as large as the specified accuracy. This suggests that the calibrations may need to be taken at closer intervals.

\section{Calibration of the Pressure Transducers}

Two situations make the wall pressure transducer calibrations more difficult to chart than the PCU calibrations. First, instead of one pressure transducer being calibrated, 384 must be analyzed; and, second, the wall pressure transducer calibrations occur daily not bi-annually.

There are fifteen different variables that must be monitored from each wall pressure transducer calibration. The first five variables are the calibration pressures output from PCU simultaneously applied to all of the transducers by the PCU. Then for each of the 384 transducers there are ten more variables, five voltages output by the transducers and five coefficients calculated from the fourth order curve fit of the calibration data. For each of these variables an individuals and moving range chart are plotted.

An example of the control charts for the five coefficients from one transducer is shown in Figures 9 through 13. The individuals and moving range charts for repeated calibrations taken during one test are shown. For all figures the chart means are shown as 
solid lines and the chart control limits are shown as light gray lines. The control limits are calculated in the same manner as those for the PCU calibration, using all of the data for the test to calculate the limits. Shown in the figures as solid symbols are points which failed the WEC rules. All of the coefficient control charts for this transducer have solid symbols, i.e. failed the WEC rules. The same was true in the other five charts for the voltages from this transducer over this test. Clearly this calibration process, for this transducer on this test, was out of control.

The number of transducer calibrations per test over the one-year time period ranged from 14 to 129 . An examination of the 34,605 individuals and moving range control charts that described the nine-test behavior of 384 transducers revealed that all transducers failed the WEC rules. External modifiers are clearly having an impact on the calibrations of the pressure transducers, throwing them out of control. That the process is out of control does not mean that the wall pressure measurements taken after a calibration are necessarily incorrect, it is only stating that there is some external influence affecting the calibration which is not accounted for. By monitoring the calibrations, in real time, the control charts may reveal these external influences while they are occurring.

Even though the standard deviations calculated from the control charts are not the normal process variation (because the process is not in statistical control), they can still reveal important conclusions. Figure 14 plots the standard deviation of the intercept coefficient, $\mathrm{C}_{0}$, over the last nine tests in the tunnel for 10 pressure transducers. During Test 508 the heater boxes, which are supposed to keep the transducers at an constant temperature, were disconnected. The effect of this action can clearly be seen in the figure. Another conclusion that can be made from Figure 14 is that the 8 th transducer has a consistently higher variation. Both conclusions may be important during data analysis and when designing future tests.

\section{Pressure Transducer Measurements}

In contrast to calibrations, which are monitored and analyzed as individual measurements, the wall pressure measurements can be analyzed in groups, yielding more information. One such test, Test 508, included grouped repeated sets of data acquired with a stand-mounted pitot static probe in the middle of the tunnel test section at $\mathrm{x}=17.75 \mathrm{ft}$. Figure 15 shows the wall signature in terms of $\mathrm{Cp}$ acquired from the transducers hooked to ceiling orifices along the eighth row. The deviations from a smooth curve along the row are predominantly due to orifice error caused by perturbations found on the walls near the orifices. In this test, eighteen $Q$ sweeps were performed in groups of three, the three sweeps occurred one after another. The $Q$ sweeps ranged from $0.5 \mathrm{psf}$ to $132 \mathrm{psf}$. Figure 15 shows the $\mathrm{Q}=57.7 \mathrm{psf}$. measurement of all 18 sweeps. The groups of sweeps were separated by other runs with different tunnel configurations. The analysis of this data reveals the dependence of the wall pressure variance on the tunnel dynamic pressure.

With grouped data there are two types of variation, within-group variation and between-group variation, with the primary difference being the time scale over which the variation is monitored. These two types of variation can be monitored with a three-way control chart. Chart 1 is a range chart which plots the maximum minus the minimum values from each group in chronological order, yielding the within-group variation. Charts 2 and 3 are the averages and the moving range charts which show between-group variation. The range chart plots chronologically the maximum minus the minimum values from each of the groups. The average chart plots chronologically the average of each group minus the grand average for the corresponding transducer. The grand average is the average of all the group averages. The moving range plots chronologically the absolute value of the difference between the previous average and the current average.

Figure 16 shows the three-way control charts for the repeated data sets that are produced from five of the 229 transducers hooked up to active orifices on the walls. For all charts the means are shown as solid lines and the chart control limits are shown as light gray lines. These transducers are connected with orifices located on the ceiling row 8 at $X=27,28,29,30,31$ feet, respectively. All of the data shown in Figure 16 were acquired when the tunnel dynamic pressure was at $\mathrm{Q}=57.7$ psf. Examining the Average Chart of Figure 16 shows that the solid first symbol of the 4th transducer failed the WEC rules for control. For this tunnel condition, only 7 of the 229 transducers that failed analysis with the WEC rules. Since the overwhelming majority of the transducers are in control, the whole process is accepted.

Results from the within-group and between-group variations must be combined to calculate the standard deviation of a single measurement taken from grouped control charts. The two important values from the transducer control charts are the average range, $\bar{R}$, and the average moving range, $\overline{m R}$. The estimated withingroup standard deviation, $\hat{\sigma}_{w g}$, is 


$$
\hat{\sigma}_{w g}=\frac{\bar{R}}{d_{2}}
$$

The quantity $d_{2}$ (equal to 1.693) is a function of the number of repeats in a group, $\mathrm{n}$, which for this data set is 3. A table for $d_{2}$ may be found in any standard SPC handbook. Accordingly, the estimated standard deviation of the group average, $\hat{\sigma}_{\bar{x}}$ is

$$
\hat{\sigma}_{\bar{x}}=\frac{\overline{m R}}{1.128}
$$

To calculate the estimated between-group standard deviation, $\hat{\sigma}_{b g}$, the estimated within-group standard deviation, $\hat{\sigma}_{w g}$, contribution is removed from the estimated standard deviation of the group average, $\hat{\sigma}_{\bar{x}}$.

$$
\hat{\sigma}_{\mathrm{bg}}=\sqrt{\hat{\sigma}_{\overline{\mathrm{x}}}{ }^{2}-\frac{\hat{\sigma}_{\mathrm{wg}}{ }^{2}}{\mathrm{n}}}
$$

The standard deviation of an individual measurement is

$$
\hat{\sigma}_{\mathrm{x}}=\sqrt{\hat{\sigma}_{\mathrm{wg}}^{2}+\hat{\sigma}_{\mathrm{bg}}^{2}}
$$

Figure 17 is a plot of the transducer standard deviation calculated for each active orifice for Test 508 with a tunnel dynamic pressure of $\mathrm{Q}=57.7 \mathrm{psf}$. The North wall orifices were not connected for this test. The dashed line in Figure 17 shows the global mean for all of the active wall orifice transducers, $\bar{\sigma}_{x}$. For this test at $Q=57.7$, calculated from the data set in $C p$, $\overline{\hat{\sigma}_{x}}=0.00114$.

Control charting the PCU calibration showed that the process is in control, though the normal process variation maybe too large. Control charting the wall pressure transducer calibrations showed that the process is not in control and is being affected by external modifiers. Control charting the wall pressure measurements for Test 508 showed that the wall pressure measurement process is in control.

\section{MONITORING SYSTEMS}

As demonstrated in the previous section, monitoring the control charts for almost 400 pressure transducer measurements can be overwhelming, thereby limiting the use of these techniques unless software tools are developed and implemented for routine application. While the SPC methods used for creating the control charts are similar, the monitoring systems required for each of the processes are quite different. Three monitoring methods have been developed and they are discussed in the following subsections.

\section{Calibration of the PCU}

Having established the process control limits based on existing data, the quality of each new PCU calibration must be compared with the historical results presented in the control charts (see Figure 8). With each added calibration the WEC Rules are evaluated to ensure the process is still operating as desired. Failure to satisfy the rules requires identification of the external modifier.

\section{Calibration of the Pressure Transducers}

Relative to the bi-annual PCU calibrations, daily calibrations of nearly 400 pressure transducers are difficult to analyze and monitor. It was demonstrated in the previous section that the pressure transducer calibration process is not in control, and that there are external modifiers effecting the calibrations. Establishing an effective monitoring system is critical to identifying and minimizing their effects.

The pressure transducer calibration monitoring program (PTCMP) is a graphical display system, setup to monitor the daily health of the wall pressure system. PTCMP is operated within the control room of the $14 \times 22 \mathrm{Ft}$. subsonic tunnel by the test engineers and technicians. The program uses SPC methods, as described in the previous section, to analyze the calibrations of the pressure transducers then display the results on a hierarchical display. The top level of the display format is the main screen, shown in Figure 18. The main screen displays a status light for each pressure transducer in the selected module. The status light reflects whether or not all 15 variables of the calibration fall within the control limits for a selected transducer. If any of the 15 measurements falls outside of the control limits on the charts, then the control light will change from green (meaning no problem) to yellow, indicating a control limit has been exceeded. If all of these quantities fall outside of the control limits, then the light will change from yellow to red. A detailed presentation of the lights for the selected port can be seen in the port display area, located on the central right side of Figure 18; port 19 is displayed in the figure. Selecting any button in the port display area will present the appropriate control charts for that port, as shown in Figure 19. Similar functionality is exhibited when using the buttons in the PCU display area, located on the central left side of Figure 18. Thus, quick look monitoring can be achieved by rapidly scanning the main screen, and detailed analysis for assessment and error tracking is readily available by appropriate selection of the color-coded lights. 
For the present setup, the individuals and moving range charts include: 5 applied pressure charts, 1,920 transducer voltages charts and 1,920 coefficient charts. The control limits used in the charts are calculated based upon first 128 calibrations saved in the database. This is rather arbitrary due to the fact that the process is not in control and therefore the limits do not represent the normal process variation. Unless the process is in control there is no normal process variation.

Based on early analysis, transducer voltages were found to drift over time. When the voltages drifted the individuals charts all fell outside of the control limits. Because the slow drift is slow, the moving range chart did not fall outside of the limits. To avoid recalculation of the limits every fifty calibrations or so, the main screen system of lights are only based upon the moving range control chart three standard deviation limits. If the drift issue is resolved, future software releases of PTCMP may re-connect the main screen warning lights to the individuals control chart.

External modifying events such as the heater boxes being turned off, are clearly visible in the control charts shown in Figure 20. Another external modifying event is shown in Figure 21 when an extreme change in the $C_{1}$ coefficient occurred. This event would be signaled by PTCMP with all control lights flashing on at the same time. Because the monitoring system was not implemented at the time, there was no chance to search for the external modification in real time. For all charts in Figures 20 and 21 the means are shown as solid lines and the chart control limits are shown as light gray lines. For both of the figures the solid symbols failed the WEC rules for control. Many other external modifications periodically affect the process, as seen in solid symbols of Figures 9 through 13; the monitoring system enables tracking them as they occur.

\section{Pressure Transducer Measurements}

PTCMP checks the day-to-day performance of the wall pressure calibration system, however this does not check the pressure measurements directly. Any test where there are repeated measurements of the same test article at the same test conditions is a candidate for monitoring the variability of the pressure transducer measurements. Test 508, discussed above, had such repeated measurements with six groups of three repeats. In another recent test, Test 506, a large semispan highlift model with a trapezoidal planform was mounted to the floor of the tunnel (see Figure. 22). The test was run at $\mathrm{Q}=57.7 \mathrm{psf}$. and through a polar of 28 angles of attack from $-4^{\circ}$ to $35^{\circ}$. Repeated data were acquired with six to ten groups of eleven runs. At the large angles of attack the model-induced wall pressures in Cp ranged from -0.10 to 0.18 . An analysis similar to that of Test 508 was performed on the data using three-way charts. The analysis showed that wall pressure measurement process in Test 506 was in control, as was Test 508. The influence on measurement variability from changing dynamic pressure as well as changing wall signature can now be compared.

Two tests of repeated measurements have been analyzed; Test 508 with nothing in the tunnel except a small Q-probe stand where the tunnel $Q$ is varied and Test 506 with a large model, swept through a large polar range, and the $Q$ held at 57.7 psf. The mean standard deviation across all of the transducers was calculated for the active orifices for each condition. Figure 23 is a plot of the mean standard deviation across all of the wall ports versus the tunnel $Q$ for Test 508. As $Q$ decreases below a value of $Q=25$ psf. the variation of the wall $C p$ values increases dramatically which can easily be seen on the semi-log scale. Wall measurements acquired at these low Q's have a significant increase in variation and may be of limited use. If additional testing is to be performed at these low $Q$ values and if computations are required that use the wall data, then further enhancements to the wall pressure measurement system to reduce this uncertainty is required.

Figure 24 shows the mean standard deviation across all of the wall ports versus the angle of attack for Test 506 . Between $2^{\circ}$ and $32^{\circ}$ the mean standard deviation is approximately constant. The value is similar to that of Figure 23 at the same Q, Q=57.7 psf. Comparing the range of $C p$ values from Test $506,-0.10$ to 0.18 , with the range from Figure 15 from Test 508, -0.001 to 0.029 , one can conclude that the magnitude of the wall signature does not have much of an effect on the variability of the wall pressure measurements. However at $34^{\circ}$ the standard deviation sharply increases. The balance data acquired for these conditions indicate that the wing entered a highly unsteady region and began to stall at $34^{\circ}$. This transition region is characterized by large fluctuating flow features about the model which did affect the variability of the wall pressure transducer measurements.

The PTCMP calibration monitoring systems enables the daily health monitoring of the wall pressure measurement system, but periodic checks of the pressure transducer measurement variability are necessary to confirm that the wall pressure measurement process is in control and that the normal variation levels have not increased. 


\section{CONCLUSIONS}

The variability of the wall pressure system was analyzed as three separate processes using Statistical Process Control charting methods. The first analysis, the calibration of the pressure calibration unit, demonstrated that this process is under control. However the normal process variation of the PCU slope adjustment was twice the prescribed value, signaling that a decrease in the time between calibrations is necessary. The second process, the calibration of the pressure transducers, is not under control, requiring a monitoring system to identify external modifiers to the process. The monitoring system subsequently was developed and installed, and is now actively being used in the tunnel. The third process, repeated measurements using the same test article, was performed on two separate sets of data. The variation in wall pressure was found to be nearly constant across a large range of dynamic pressure and model lift coefficient; however, at low tunnel dynamic pressures and conditions generating fluctuating flow features the variability increased. The demonstrated increased variation at low dynamic pressures may warrant further improvements to the pressure system for these test conditions. Periodic monitoring of repeated data sets is necessary for confirming that the wall pressure measurement process is in control and that the normal process variations are within established process limits.

Long-term monitoring is the key to maintaining the data quality standards of the 400 transducer readings in this complex wall pressure data system. When used systematically, Statistical Process Control methods provide the necessary tools to measure and control the variability.

\section{REFERENCES}

1. Walker, E., Everhart, J., and Iyer, V., "Sensitivity Study of the Wall Interference Correction System (WICS) for Rectangular Tunnels," AIAA Paper 20010159, January 2001 .

2. Anon., Manual on Presentation of Data and Control Chart Analysis: $6^{\text {th }}$ Edition, ASTM Manual Series: MNL7, 1995.

3. Croarkin, Carroll, and Tobias, Paul (Eds.), NIST/Sematech Engineering Statistics Handbook. (online)

http://www.itl.nist.gov/div898/handbook/main.htm. Accessed June 3, 2003.

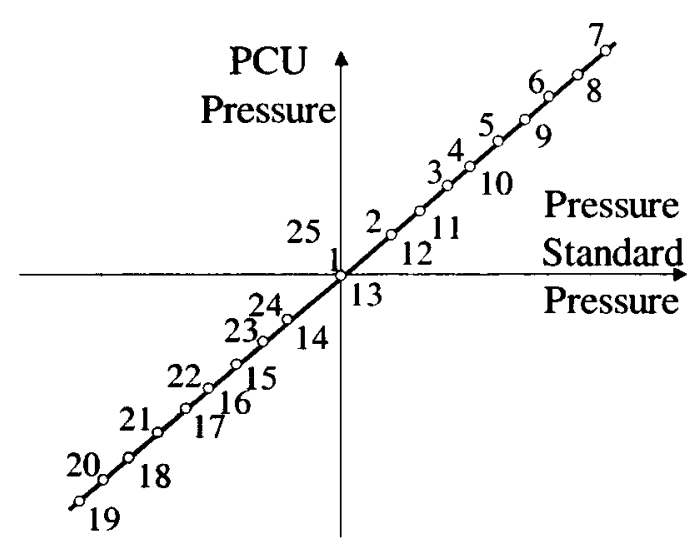

Figure 1. Typical curve of the PCU calibration, sequenced in the order shown.

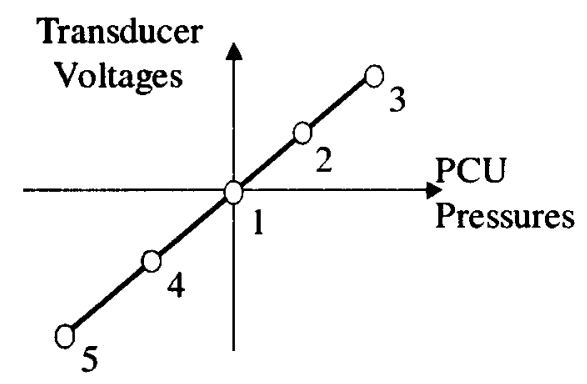

Figure 2. Typical curve of the pressure module calibration, sequenced in the order shown.

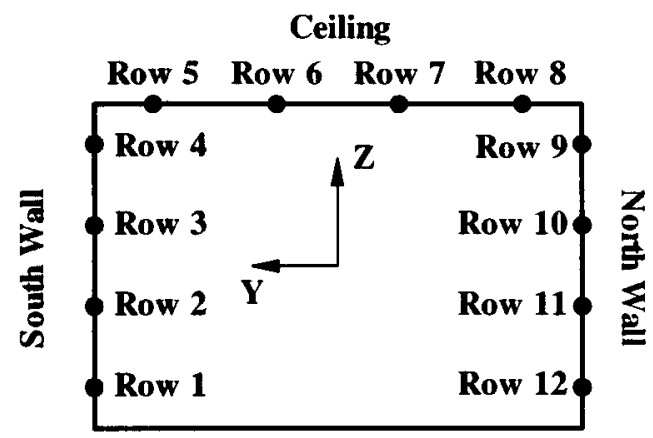

Looking Downstream

Figure 3. Wall orifice row locations in the NASA Langley 14x22 Ft Subsonic Tunnel. 


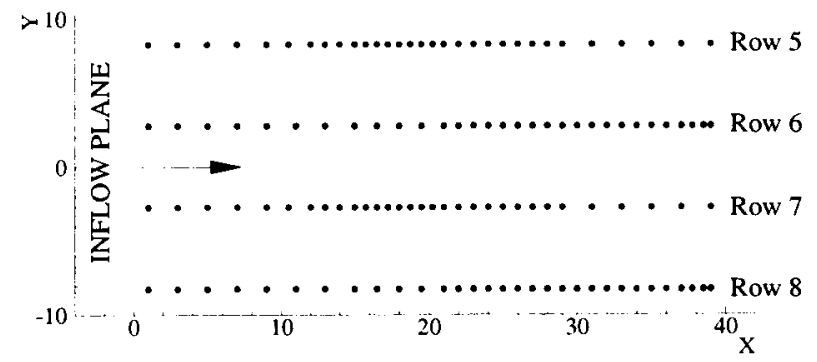

Figure 4. Orifice layout on ceiling of $14 \times 22 \mathrm{Ft}$ Subsonic Tunnel.

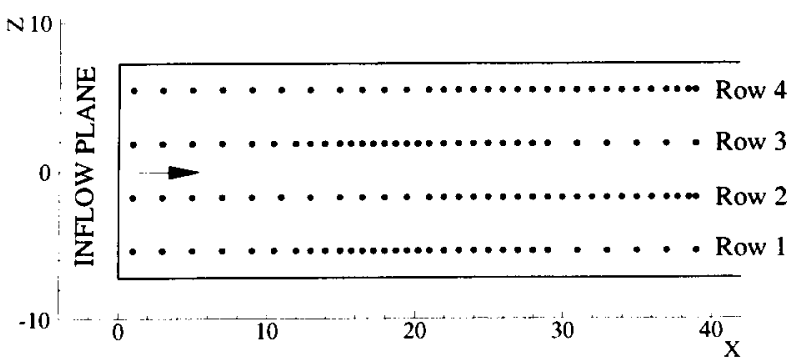

Figure 5. Orifice layout on South wall side view of $14 \times 22 \mathrm{Ft}$ Subsonic Tunnel.

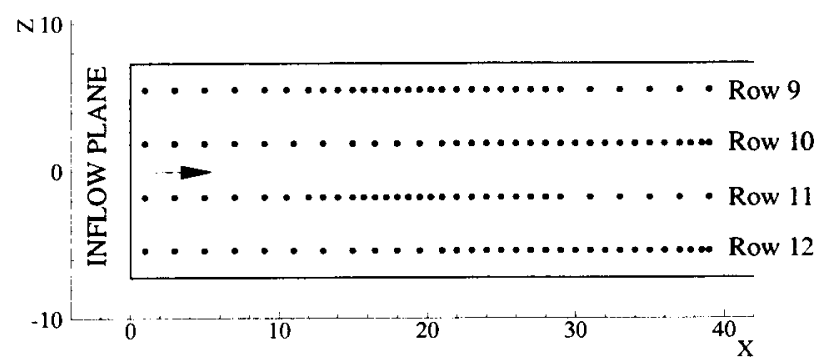

Figure 6. Orifice layout on North wall of $14 \times 22 \mathrm{Ft}$ Subsonic Tunnel.

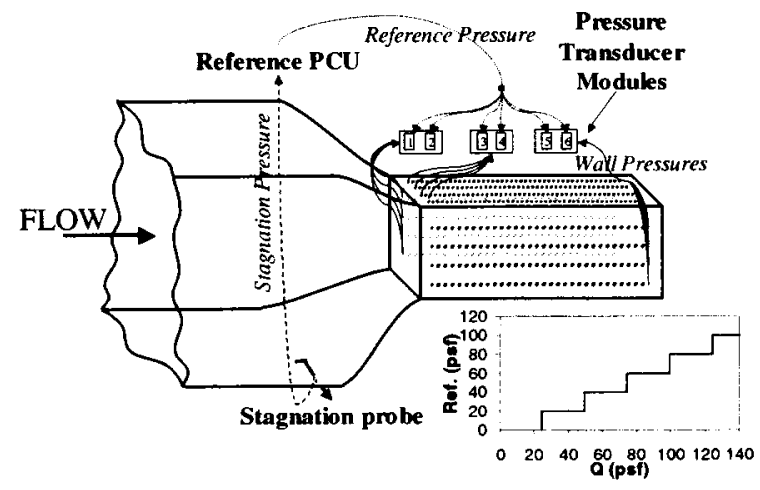

Figure 7. Schematic of the reference pressure system for Pressure Transducer Modules.

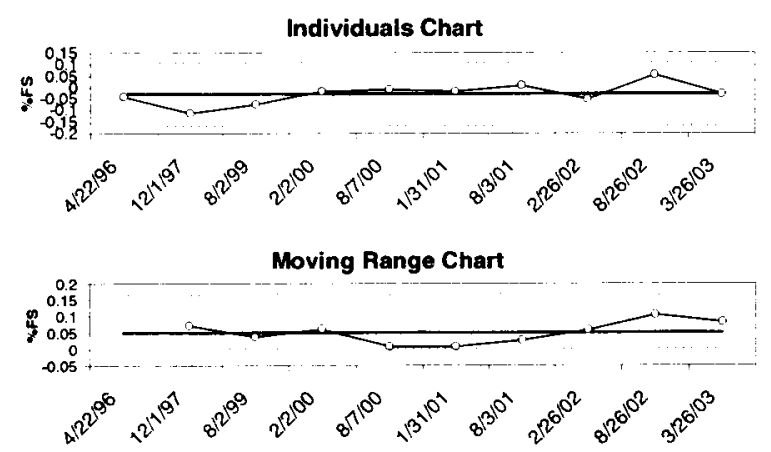

Figure 8. The slope adjustment PCU calibration control chart. The $\mathrm{Y}$-axis are the slope adjustment values in $\%$ FS of 1 psid. The $\mathrm{X}$-axis is the calibration date.

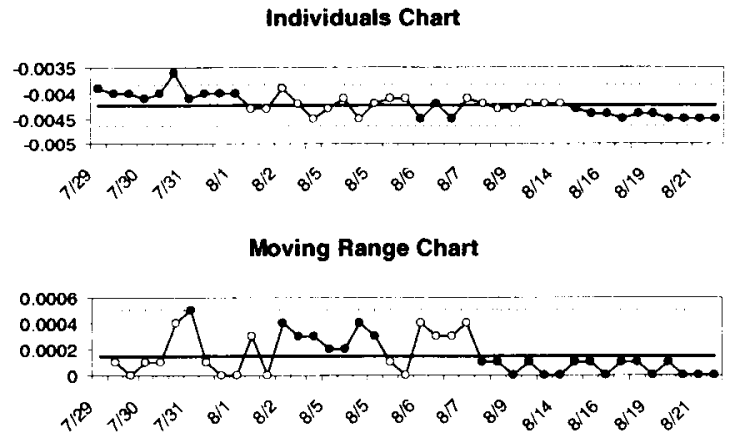

Figure $9 . \mathrm{C}_{0}$ coefficient calibration control chart across Test 506 for one pressure transducer. The $\mathrm{Y}$-axis is the $\mathrm{C}_{0}$ coefficient calibration values; $\mathrm{X}$-axis is the calibration date.

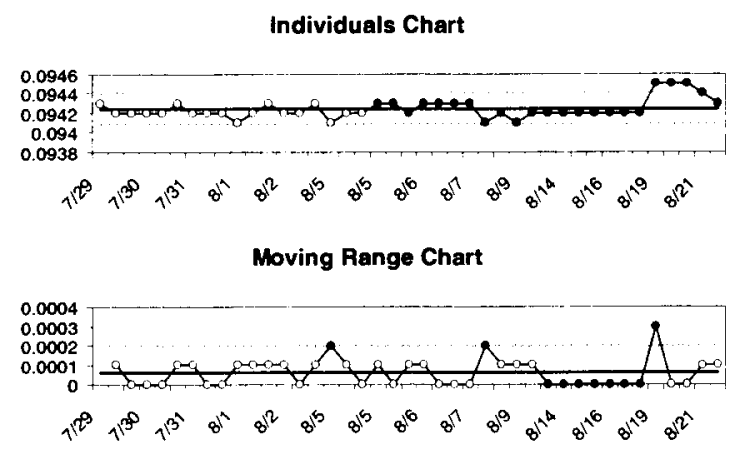

Figure 10. $\mathrm{C}_{1}$ coefficient calibration control chart across Test 506 for one pressure transducer. The $\mathrm{Y}$ axis is the $C_{1}$ coefficient calibration values; $X$-axis is the calibration date. 


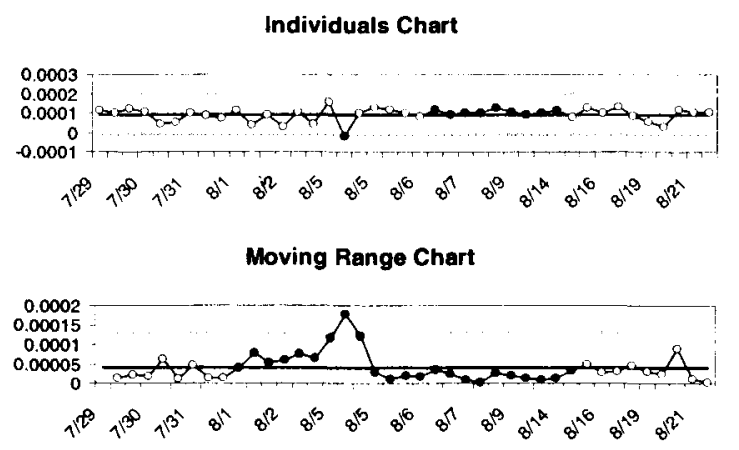

Figure 11. $\mathrm{C}_{2}$ coefficient calibration control chart across Test 506 for one pressure transducer. The Yaxis is the $\mathrm{C}_{2}$ coefficient calibration values; $\mathrm{X}$-axis is the calibration date.

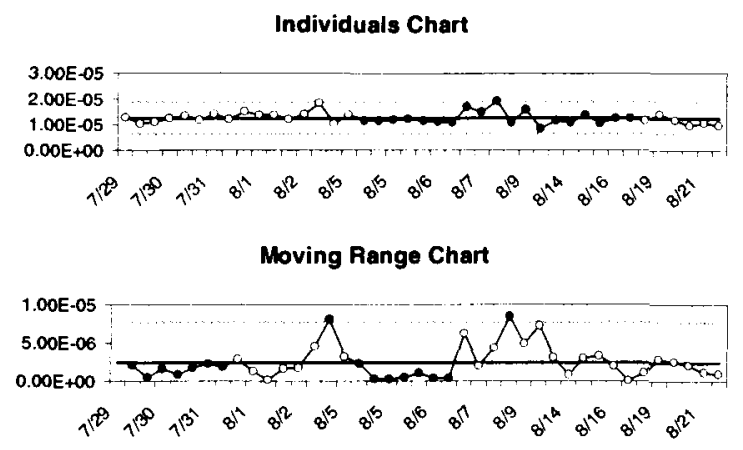

Figure 12. $\mathrm{C}_{3}$ coefficient calibration control chart across Test 506 for one pressure transducer. The Yaxis is the $\mathrm{C}_{3}$ coefficient calibration values; $\mathrm{X}$-axis is the calibration date.

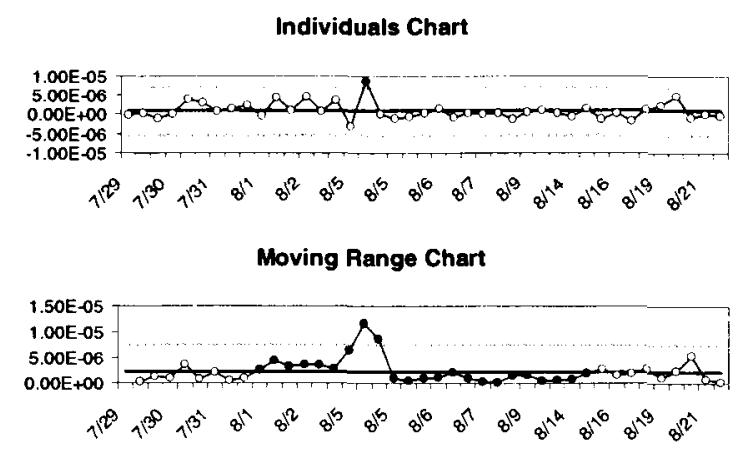

Figure 13. $\mathrm{C}_{4}$ coefficient calibration control chart across Test 506 for one pressure transducer. The Yaxis is the $\mathrm{C}_{4}$ coefficient calibration values; $\mathrm{X}$-axis is the calibration date.

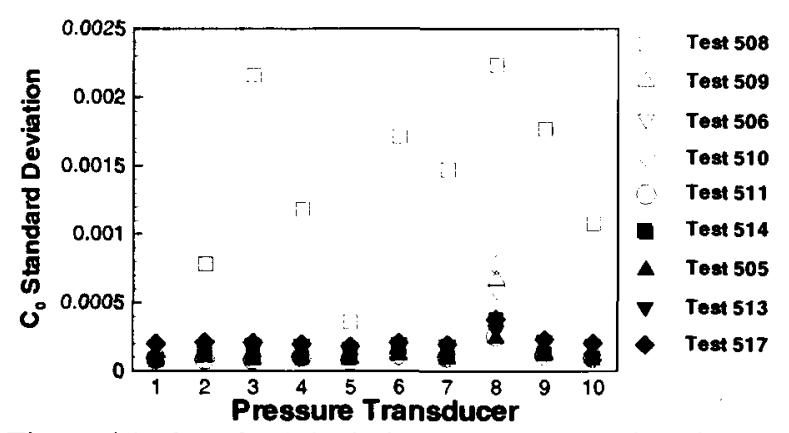

Figure 14. Standard deviations of the $\mathrm{C}_{0}$ Calibration coefficients calculated over nine tests for 10 pressure transducers.

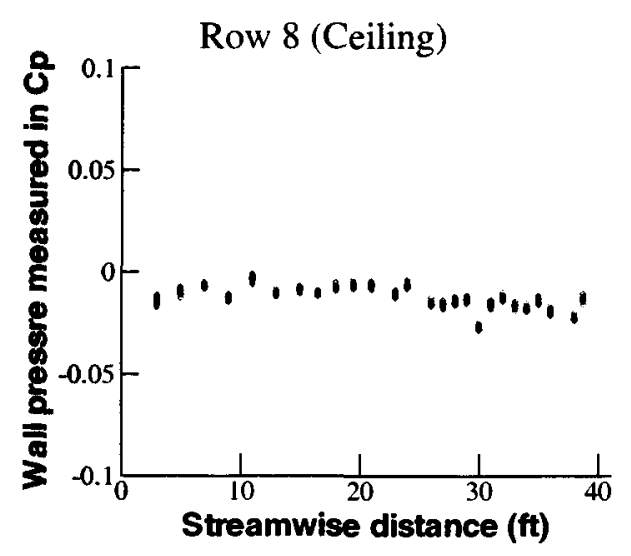

Figure 15. Wall pressure measurement signature for Test 508, Row 8, $Q=57.7$, eighteen runs plotted.
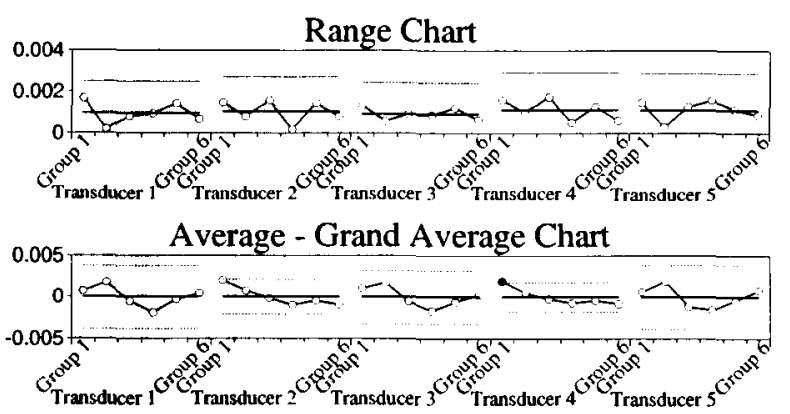

Moving Range Chart

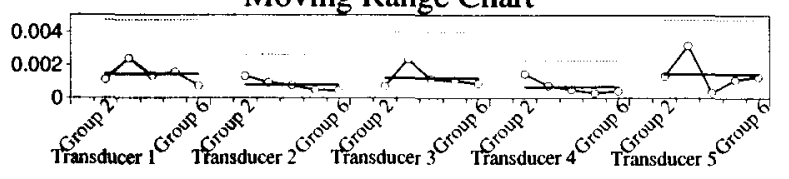

Figure 16. Three-way Control charts for selected pressure transducers from Test $508, \mathrm{Q}=57.7 \mathrm{psf}$; Groups: 1, (Runs 201, 202, 203); 2, (207, 208, 209); 3, (215, 216, 217); 4, (251, 252, 253); 5, (258, 259, 260); $6,(264,265,266)$. The $Y$-axis is the wall pressures measured in $\mathrm{Cp}$; $\mathrm{X}$-axis are the groups of runs for five different transducers. 

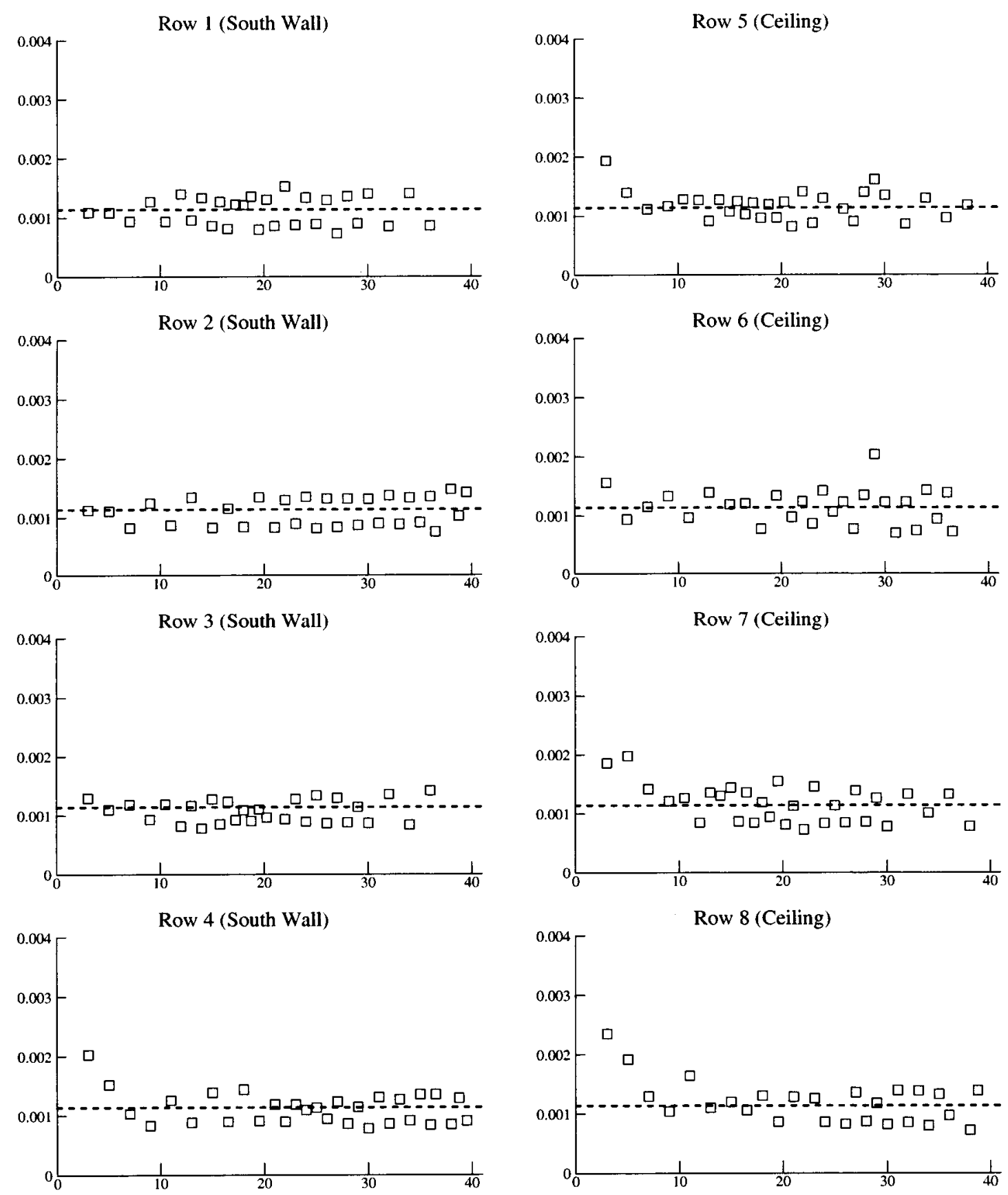

Figure 17. Transducer standard deviations for Test 508 , from $\mathrm{Cp}$ wall pressure measurements at $\mathrm{Q}=57.7$ psf. The dashed line is the mean calculated across all of the active wall orifice transducers, $\overline{\hat{\sigma}}_{\mathrm{x}}$. The $\mathrm{Y}$ axis is the standard deviation of the wall pressures measured in $\mathrm{Cp}$; $\mathrm{X}$-axis is the streamwise distance $\mathrm{X}$ in feet. 


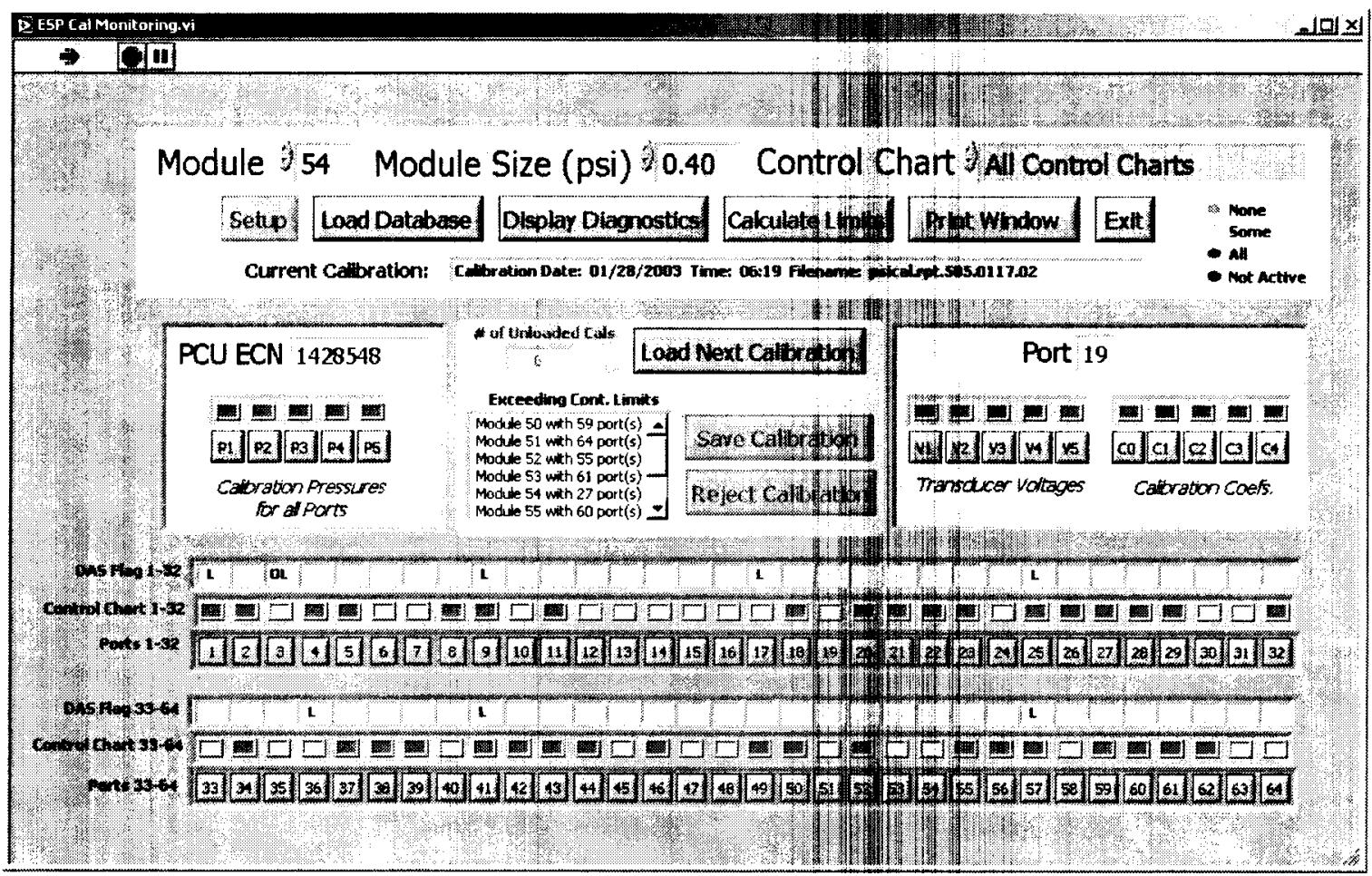

Figure 18. Main display screen for pressure transducer calibration monitoring program.

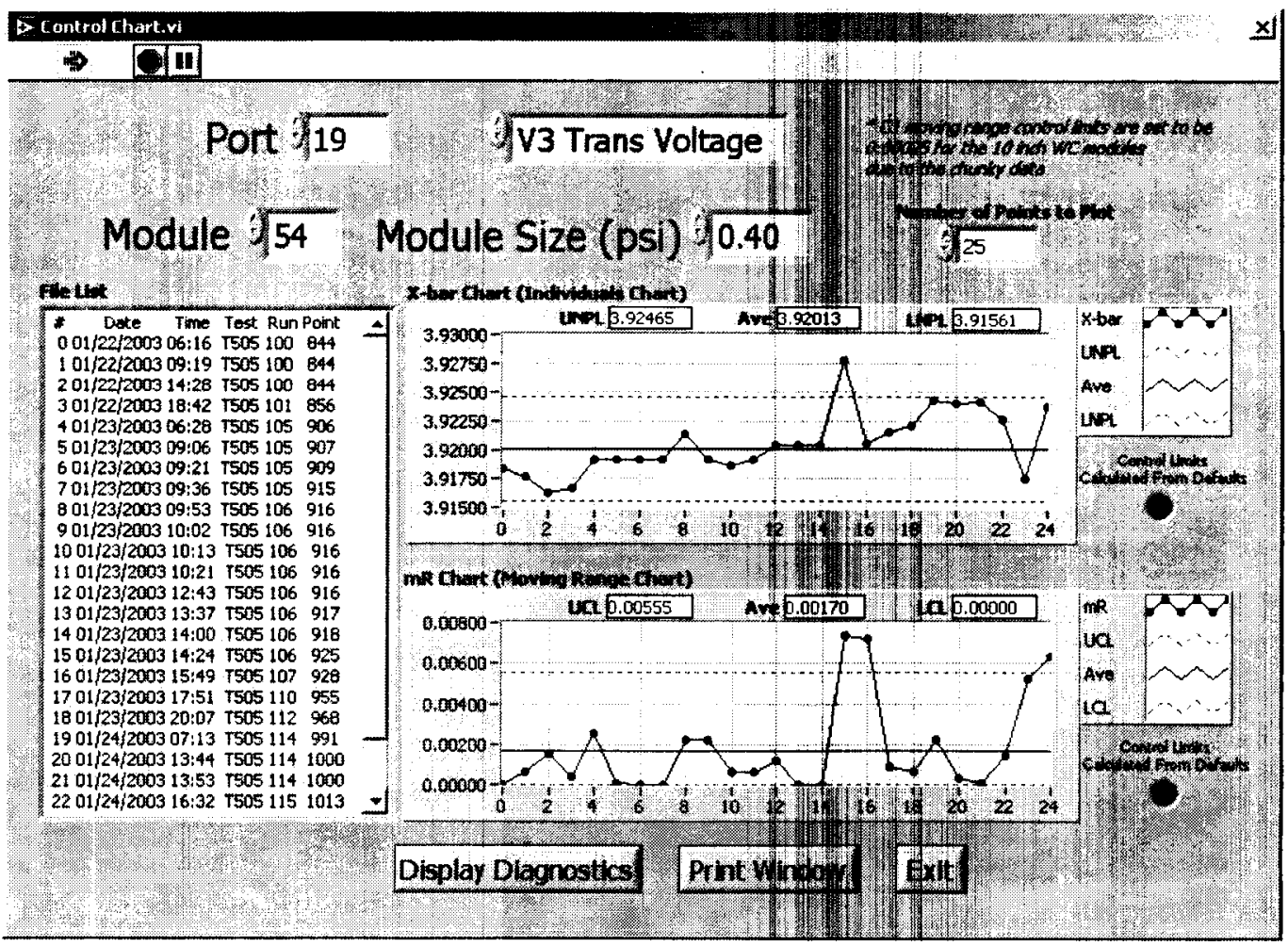

Figure 19. Control chart screen for pressure transducer calibration monitoring program. 


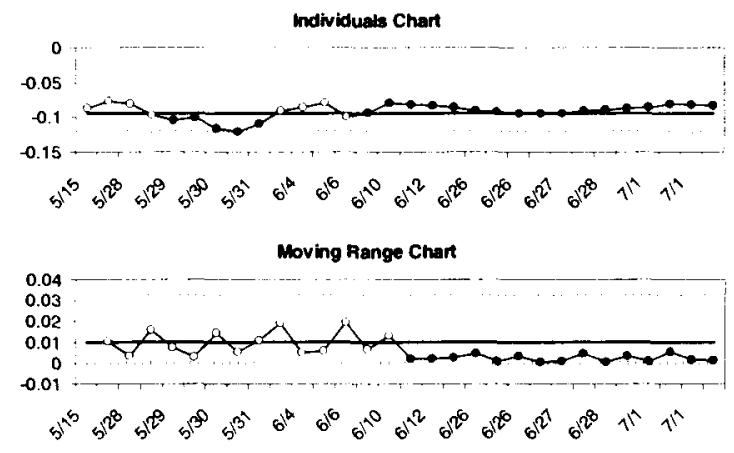

Figure 20. Use of Moving Range Charts to identify measurement system proglems. Heater control box turned on for calibrations taken on and before 6/10/2002; heater control boxes off afterwards. Control limits calculated using data on and before 6/10/2002. The $\mathrm{Y}$-axis is the $0 \mathrm{psi}$. transducer voltage values; $\mathrm{X}$ axis is the calibration date.

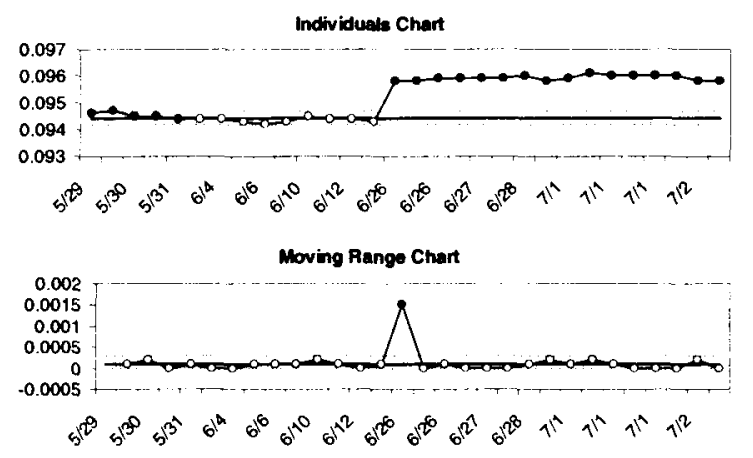

Figure 21. Use of Individuals and Moving Range Charts to identify transducer calibration problems. $\mathrm{Cl}$ coefficient jump occurred between 6/13/2002 and $6 / 26 / 2002$, control limits are calculated based upon data on and before 6/13/2002. The $\mathrm{Y}$-axis is the $\mathrm{C}_{1}$ coefficient calibration values; $\mathrm{X}$-axis is the calibration date.

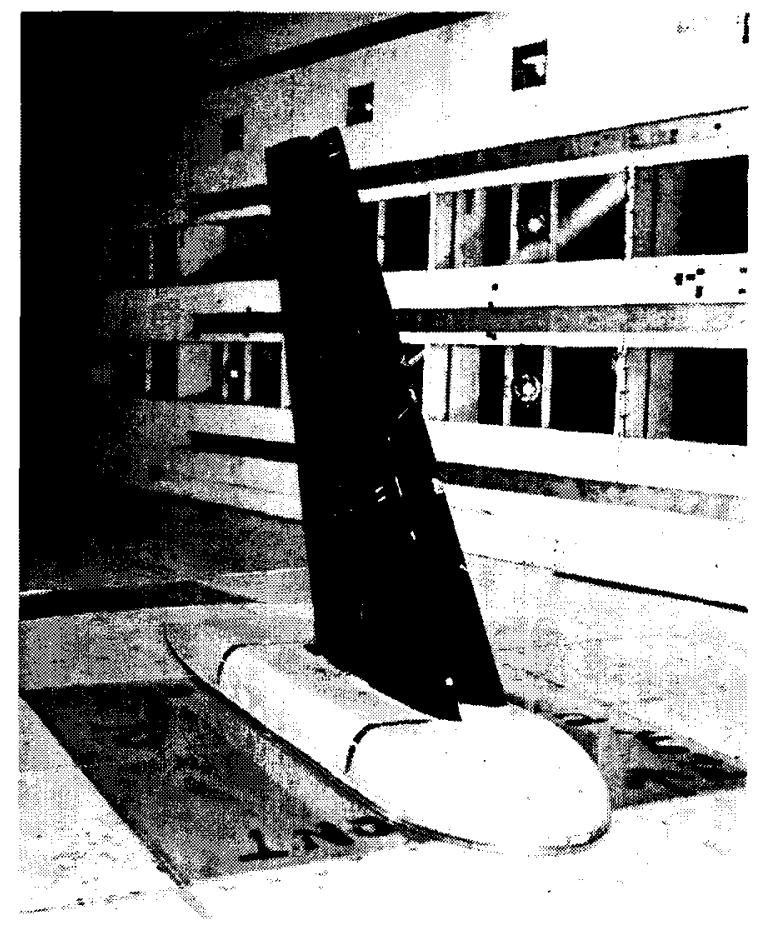

Figure 22. Photo of the trapezoidal wing mounted in the tunnel. Photo courtesy of NASA Langley Photo Lab.

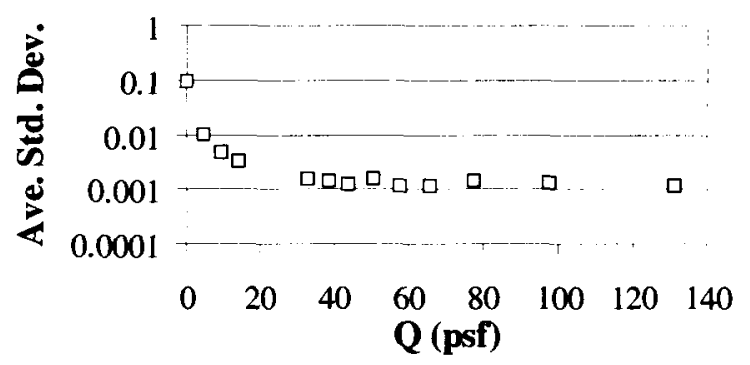

Figure 23. Test 508 mean standard deviation variation with dynamic pressure in the tunnel.

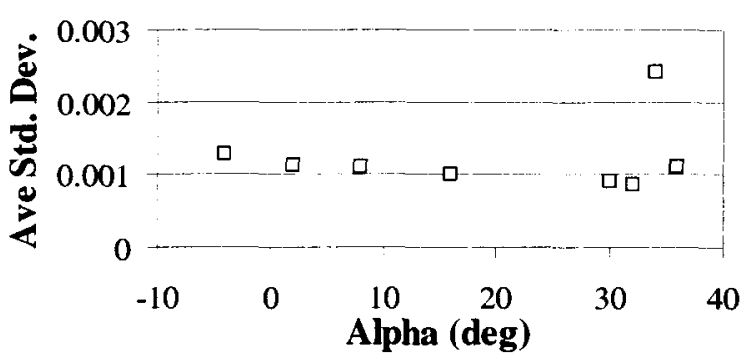

Figure 24. Test 506 mean standard deviation variation with angle of attack of model in the tunnel with a tunnel dynamic pressure of $\mathrm{Q}=57.7 \mathrm{psf}$. 